\begin{tabular}{c} 
International Journal of Applied Mathematical Research, $7(1)(2018) 20-26$ \\
International Journal of Applied Mathematical Research \\
Website: $\begin{array}{c}\text { www.sciencepubco.com/index.php/IJAMR } \\
\text { doi: } 10.14419 / \text { ijamr.vil. } 8836 \\
\text { Research paper }\end{array}$ \\
\hline
\end{tabular}

\title{
A Class of new exact solutions of Navier-Stokes equations with body force for viscous incompressible fluid
}

\author{
Mushtaq Ahmed $^{1 *}$, R. K. Naeem ${ }^{2}$ and S. Anwer Ali ${ }^{3}$ \\ ${ }^{1}$ Department of Mathematics, University of Karachi,, Karachi-75270, Pakistan, \\ ${ }^{2}$ Department of Mathematics, University of Karachi,, Karachi-75270, Pakistan, \\ ${ }^{3}$ Department of Mathematics, University of Karachi,, Karachi-75270, Pakistan, \\ *mushtaqku@yahoo.com,muahmed@uok.edu.pk
}

\begin{abstract}
This paper is to indicate a class of new exact solutions of the equations governing the two-dimensional steady motion of incompressible fluid of variable viscosity in the presence of body force. The class consists of the stream function $\psi$ characterized by equation $\theta=f(r)+a \psi+b$ in polar coordinates $r, \theta$, where a continuously differentiable function is $f(r)$ and $a \neq 0, b$ are constants. The exact solutions are determined for given one component of the body force, for both the cases when $f(r)$ is arbitrary and when it is not. When $f(r)$ is arbitrary, we find $a=1$ and we can construct an infinite set of streamlines and the velocity components, viscosity function, generalized energy function and temperature distribution for the cases when $R_{e} P_{r}=1$ and when $R_{e} P_{r} \neq 1$ where $R_{e}$ represents Reynolds number and $P_{r}$ Prandtl number. For the case when $f(r)$ is not arbitrary we can find solutions for the cases $R_{e} P_{r} \neq a$ and $R_{e} P_{r}=a$ where " $a$ " remains arbitrary.
\end{abstract}

Keywords: Exact solutions to the flow equations of incompressible fluids; Variable viscosity fluids; Navier-Stokes equations with body force; Exact solutions in the presence of body force; Martin's coordinates system.

\section{Introduction}

A moving fluid element experiences forces, directly on its volumetric mass as well as on its surface, named body forces and surface forces respectively. The examples of body forces are gravitational force, electric force or coriolis force etc. In a fluid flow model, we keep the product of mass and acceleration of the moving fluid element in lefthand side and net forces on it in right-hand side of the momentum equation known as Navier-Stokes equations (NSE). The complex mathematical structure of NSE offers a great difficulty in achieving exact solutions; however, we find some transformation techniques and dimension analysis method that were helpful in providing some new exact solutions. We refer here [1-13] and reference therein for some exact solutions of NSE with surface forces on right-hand side of NSE using a variety of techniques/methods, however [14] considered NSE with coriolis force and [15] gave a basic remark on NSE with body force. We further mention here some attempts to the problem of finding exact solutions of the equations describing the steady plane flows of incompressible fluid of variable viscosity in the presence of body force. Naeem R. K., Aurnangzeb et. al. [16] made an effort for exact solutions of the problem mentioned above with a new coordinate transformations technique but their technique pressed them to drop the body force term at the end. Naeem R. K., Razia Shaheen [17] tried the same problem but their technique forced them to relax the variable viscosity condition.

Since here we want to keep the viscosity variable and body force therefore, we apply the technique used in Mushtaq A. [18], planned by Naeem R. K., which had successfully applied for exact solutions of the equations describing the steady plane flows of incompressible fluid of variable viscosity in the absence of body force [19]. To achieve the aim of this communication we transform the basic flows equations in Cartesian space $(x, y)$ into a curvilinear coordinates $(\varphi, \psi)$ and follow Martin [20] where he defined the coordinate lines $\psi=$ const. as streamlines and left the coordinate lines $\varphi=$ const . are arbitrary. We will be calling this $(\varphi, \psi)$ - system as Martin's system. As the coordinate $\varphi$ is arbitrary in Martin's system, therefore, we take $\varphi=r(x, y)$ to achieve our plan and we characterize the streamlines of the class of flows under consideration by

$$
\frac{\theta-f(r)-b}{a}=\text { const }
$$

where $f(r)$ is a continuously differentiable function, $r, \theta$ the polar coordinates and $a \neq 0 b$ are constants. The equation (1) implies

$\theta=f(r)+v(\psi)$

where $v(\psi)=a \psi+b$

We organize this paper as follow: In section (2), we give basic flow equations in non-dimensional form and transform them into Martin's system in section (3). In section (4), we find exact solution taking $\varphi=r(x, y)$. In section (5), we present conclusions.

\section{Non-dimensional basic flow equations}

Fluid flow with variable viscosity comprises of equation of continuity, Navier-Stokes equations and energy equation. These equations, 
for the steady plane motion of incompressible fluid of variable viscosity with constant thermal conductivity in the presence of unknown external force with no heat addition, in non-dimensional form are respectively following

$$
u_{x}+v_{y}=0
$$

$$
\begin{gathered}
u u_{x}+v u_{y}=F_{1}-p_{x}+\frac{1}{R_{e}}\left\lceil\left(2 \mu u_{x}\right)_{x}+\left\{\mu\left(u_{y}+v_{x}\right)\right\}_{y}\right\rceil \\
u v_{x}+v v_{y}=F_{2}-p_{y}+\frac{1}{R_{e}}\left\lceil\left(2 \mu v_{y}\right)_{y}+\left\{\mu\left(u_{y}+v_{x}\right)\right\}_{x}\right\rceil \\
u T_{x}+v T_{y}=\frac{1}{R_{e} P_{r}}\left(T_{x x}+T_{y y}\right) \\
+\frac{E_{c}}{R_{e}}\left\lceil 2 \mu\left(u_{x}^{2}+v_{y}^{2}\right)+\mu\left(u_{y}+v_{x}\right)^{2}\right\rceil
\end{gathered}
$$

Where $u, v$ are the components of velocity, $F_{1}, F_{2}$ are the components of the body force, $\mu$ the viscosity, $p$ pressure and $T$ is temperature. All these seven quantities are function of $x$ and $y$. The numbers $R_{e}, E_{c}$ and $P_{r}$ are the Reynolds number, the Ecart number and the Prandtl number respectively.

The solution of the equation (3) of continuity provides a stream function $\psi(x, y)$ such that

$u=\psi_{y}, v=-\psi_{x}$

Experience shows that the exact solution of equations (4-6) offers a great difficulty because of the presence of the non-linear term, therefore we write equation (4-6) in following manage able form by introducing the vorticity function $w$ and the total energy function $L$ defined by

$w=v_{x}-u_{y}$

$L=p+\frac{1}{2}\left(u^{2}+v^{2}\right)-\frac{2 \mu u_{x}}{R_{e}}$

Differentiating equation (9) with respect to $x$ and substituting the resulting equation into equation (4), we get

$$
\left.-v w=F_{1}-L_{x}+\frac{\mu\left(u_{y}\right.}{+} v_{x}\right) R_{e}
$$

Similarly differentiating equation (9) with respect to $y$ and substituting the resulting equation into equation (5), we get

$u w=F_{2}-L_{y}-\frac{\left(4 \mu u_{x}\right)_{y}}{R_{e}}+\frac{\left(\mu\left(u_{y}+v_{x}\right)\right)_{x}}{R_{e}}$

Introducing further

$A=\mu\left(u_{y}+v_{x}\right), B=4 \mu u_{x}$

Substituting equation (12) in equations (10-11), we have

$-v w=F_{1}-L_{x}+\frac{A_{y}}{R_{e}}$ $u w=F_{2}-L_{y}-\frac{B_{y}}{R_{e}}+\frac{A_{x}}{R_{e}}$

Utilizing equation (12) we find the second term on the right-hand side of the equation (6) becomes $\frac{\left(B^{2}+4 A^{2}\right)}{4 \mu}$. Therefore, equation (6) gives

$u T_{x}+v T_{y}=\frac{\left(T_{x x}+T_{y y}\right)}{R_{e} P_{r}}+\frac{E_{c}\left(B^{2}+4 A^{2}\right)}{4 R_{e} \mu}$

\section{Transforming Basic flow equations into Mar- tin's system}

Let us now introduced here a curvilinear coordinate system $(\varphi, \psi)$ in the $(x, y)$-plane through transformation

$x=x(\varphi, \psi), y=y(\varphi, \psi)$

such that the Jacobian, $J=\frac{\partial(x, y)}{\partial(\varphi, \psi)}$ of the transformation is non-zero and finite.

Now following Martin [19], we define the function $\psi(x, y)$ in (16) as the stream function and leave the curves $\varphi(x, y)=$ const . as arbitrary thus the first fundamental form in Martin's system is

$d s^{2}=E(\varphi, \psi) d \varphi^{2}+2 F(\varphi, \psi) d \varphi d \psi+G(\varphi, \psi) d \psi^{2}$

wherein

$E=x_{\varphi}^{2}+y_{\varphi}^{2}, F=x_{\varphi} x_{\psi}+y_{\varphi} y_{\psi}, G=x_{\psi}^{2}+y_{\psi}^{2}$

Differentiating equation (16) with respect to $x$ and $y$, and solving the resulting equations, we find

$y_{\varphi}=-J \psi_{x}, y_{\psi}=J \varphi_{x}, x_{\varphi}=J \psi_{y}, x_{\psi}=-J \phi_{y}$

wherein

$J= \pm \sqrt{E G-F^{2}}= \pm\left(x_{\varphi} y_{\psi}-y_{\varphi} x_{\psi}\right)= \pm W$

At a point $P(x, y)$, let $\alpha$ be the angle between the tangent to the coordinate lines $\psi=$ const . and the curves $\varphi=$ const. then we find

$\tan (\alpha)=\frac{y_{\varphi}}{x_{\varphi}}$

Applying trigonometric identities and equation (18), we get

$$
\begin{gathered}
x_{\varphi}=\sqrt{E} \cos (\alpha), x_{\psi}=\frac{1}{\sqrt{E}}[F \cos (\alpha)-J \sin (\alpha)], \\
y_{\varphi}=\sqrt{E} \sin (\alpha), y_{\psi}=\frac{1}{\sqrt{E}}[F \sin (\alpha)+J \cos (\alpha)]
\end{gathered}
$$

The integribility conditions

$x_{\varphi \psi}=x_{\psi \varphi}, y_{\varphi \psi}=y_{\psi \varphi}$,

for $x$ and $y$, yield

$\alpha_{\varphi}=\frac{J \Gamma_{11}^{2}}{E}, \alpha_{\psi}=\frac{J \Gamma_{12}^{2}}{E}$

wherein 


$$
\begin{array}{r}
\Gamma_{11}^{2}=\frac{1}{2 W^{2}}\left[-F E_{\varphi}+2 E F_{\varphi}-E E_{\psi}\right] \\
\Gamma_{12}^{2}=\frac{1}{2 W^{2}}\left[E G_{\varphi}-F E_{\psi}\right]
\end{array}
$$

The equation (21), applying the integrability condition $\alpha_{\varphi \psi}=\alpha_{\psi \varphi}$ for $\alpha(\varphi, \psi)$, yields

$K=\frac{1}{W}\left[\left(\frac{W \Gamma_{11}^{2}}{E}\right)_{\psi}-\left(\frac{W \Gamma_{12}^{2}}{E}\right)_{\varphi}\right]$

where $K$ is called the Gausian curvature and equation (26) is called Gaussian equation. This equation represents a necessary condition that $E(\varphi, \psi), F(\varphi, \psi)$ and $G(\varphi, \psi)$ are coefficients of the first fundamental form in equation (18).

Now equations (13-14) on substituting equation (19), equation (22) and equations (24-25) simplifies as follow

$$
\begin{array}{r}
-R_{e} w J E=R_{e} J \sqrt{E}\left\lceil-F\left(F_{1} \cos \alpha+F_{2} \sin \alpha\right)\right. \\
\left.+J\left(F_{1} \sin \alpha-F_{2} \cos \alpha\right)\right\rceil+R_{e} J E L_{\psi} \\
+A_{\varphi}\left(\left(F^{2}-J^{2}\right) \cos 2 \alpha-2 F J \sin 2 \alpha\right) \\
+E A_{\psi}(J \sin 2 \alpha-F \cos 2 \alpha) \\
-B_{\varphi}\left\lceil\frac{1}{2}\left(\left(F^{2}-J^{2}\right) \sin 2 \alpha+F J \sin 2 \alpha\right\rceil\right. \\
+E B_{\psi}\left(\frac{1}{2} F \sin 2 \alpha+J \cos ^{2} \alpha\right)
\end{array}
$$

and

$$
\begin{array}{r}
\left.0=R_{e} J \sqrt{E}\left\lceil F_{1} \cos \alpha+F_{2} \sin \alpha\right)\right\rceil-R_{e} J L_{\varphi} \\
+E A_{\psi} \cos 2 \alpha-A_{\varphi}\lceil F \cos 2 \alpha-J \sin 2 \alpha) \\
+B_{\varphi}\left\lceil\frac{1}{2} F \sin 2 \alpha-J \sin ^{2} \alpha\right\rceil-\frac{E B_{\psi}}{2} \sin 2 \alpha
\end{array}
$$

Differential geometry [21] says that

$$
T_{x x}+T_{y y}=\frac{1}{J}\left[\left(\frac{G T_{\varphi}-F T_{\psi}}{J}\right)_{\varphi}+\left(\frac{E T_{\psi}-F T_{\varphi}}{J}\right)_{\psi}\right]
$$

and the expression $u T_{x}+v T_{y}$ in the left-hand side of equation (15) simplifies to $\frac{T_{\varphi}}{J}$, thus the energy equation (15) becomes

$$
\begin{array}{r}
\frac{1}{J R_{e} P_{r}}\left[\left(\frac{G T_{\varphi}-F T_{\psi}}{J}\right)_{\varphi}+\left(\frac{E T_{\psi}-F T_{\varphi}}{J}\right)_{\psi}\right] \\
=-\frac{E_{c}\left(B^{2}+4 A^{2}\right)}{4 \mu R_{e}}+\frac{T_{\varphi}}{J}
\end{array}
$$

Let the magnitude of velocity vector $\mathbf{q}=(u, v)$ is $q=\sqrt{u^{2}+v^{2}}$, then substituting values from equation (18-19), we find

$q=\frac{\sqrt{E}}{J}$

The equation (12) on substituting equation (19), (22) and equations (24-25), provides

$$
\begin{array}{r}
A(\varphi, \psi)=\mu\left\lceil-\frac{(F \cos \alpha-J \sin \alpha)}{4 E^{2} J^{5}}\right. \\
\left\{E_{\varphi}\left(2 E J^{3} \cos \alpha+F \sqrt{E} \sin \alpha\right)-4 E^{2} J^{2} J_{\varphi} \cos \alpha\right. \\
\left.-2 E \sqrt{E} F_{\varphi} \sin \alpha+E \sqrt{E} E_{\psi} \sin \alpha\right\} \\
+\frac{\cos \alpha}{2 J^{3}}\left\{E_{\psi}(F \sin \alpha+J \cos \alpha)\right. \\
\left.-2 E J_{\psi} \cos \alpha-E G_{\varphi} \sin \alpha\right\} \\
+\frac{(F \sin \alpha+J \cos \alpha)}{2 E J^{3}}\left\{\left(J E_{\varphi}-2 E J_{\varphi}\right) \sin \alpha\right. \\
\left.+\cos \alpha\left(-F E_{\varphi}+2 E F_{\varphi}-E E_{\psi}\right)\right\} \\
-\frac{\sin \alpha}{2 J^{3}}\left\{E_{\psi}(J \sin \alpha-F \cos \alpha)\right. \\
\left.\left.-2 E J_{\psi} \sin \alpha+E G_{\varphi} \cos \alpha\right\}\right\rceil
\end{array}
$$

and

$$
\begin{array}{r}
B(\varphi, \psi)=\frac{4 \mu}{E J^{3}}\left\lceil E_{\varphi}(F \sin \alpha+J \cos \alpha)^{2}\right. \\
+E^{2}\left(J_{\psi} \sin 2 \alpha+G_{\varphi} \sin ^{2} \alpha\right) \\
\left.-2 E(F \sin \alpha+J \cos \alpha)\left(F_{\varphi} \sin \alpha+J_{\varphi} \cos \alpha\right)\right\rceil
\end{array}
$$

Equation (8) in Martin's system is

$w=v_{\varphi} \varphi_{x}+v_{\psi} \psi_{x}-u_{\varphi} \varphi_{y}-u_{\psi} \psi_{y}$

Substituting equation (19), (22) and equations (24-25), and equation (31), we find

$$
\begin{array}{r}
w=\frac{(F \sin \alpha+J \cos \alpha)}{2 E J^{3}}\left\lceil\left(J E_{\varphi}-2 E J_{\varphi}\right) \sin \alpha\right. \\
\left.+\cos \alpha\left(-F E_{\varphi}+2 E F_{\varphi}-E E_{\psi}\right)\right\rceil \\
-\frac{\sin \alpha}{2 J^{3}}\left\lceil E_{\psi}(J \sin \alpha-F \cos \alpha)\right. \\
\left.-2 E J_{\psi} \sin \alpha+E G_{\varphi} \cos \alpha\right\rceil \\
+\frac{(F \cos \alpha-J \sin \alpha)}{4 E^{2} J^{5}}\left\lceil E_{\varphi}\left(2 E J^{3} \cos \alpha+F \sqrt{E} \sin \alpha\right)\right. \\
\left.-4 E^{2} J^{2} J_{\varphi} \cos \alpha-2 E \sqrt{E} F_{\varphi} \sin \alpha+E \sqrt{E} E_{\psi} \sin \alpha\right\rceil \\
-\frac{\cos \alpha}{2 J^{3}}\left\lceil E_{\psi}(F \sin \alpha+J \cos \alpha)\right. \\
\left.-2 E J_{\psi} \cos \alpha-E G_{\varphi} \sin \alpha\right\rceil
\end{array}
$$

Thus, the basic system of non-dimensional partial differential equations governing steady plane flow of an incompressible fluid of variable viscosity, in the presence of external force with no heat addition are transformed into Martin's system as equations (31), (27-30), (32-33) and (35).

\section{Exact Solutions}

Since our objective is to determine a class of exact solution to flow equations for which the streamlines are characterizes by equation (2) and the coordinate $\varphi$ is arbitrary in Martin's system therefore we set

$\varphi=r(x, y)$

where

$x=r \cos \alpha, y=r \sin \alpha$

Utilizing equation (36-37) in equations (31), (27-30), (32-33) and (35), we get 


$$
\begin{gathered}
q=\frac{\sqrt{1+M^{2}}}{a r}, M(r)=r f^{\prime}(r) \\
-R_{e} w=-R_{e}\left(a r F_{2}\right)+R_{e} L_{\psi}-a r A_{r} \\
+M A_{\psi}+B_{\psi} \\
0=R_{e}\left(F_{1}+M F_{2}\right)-R_{e} L_{r}+\frac{A_{\psi}\left(1-M^{2}\right)}{a r} \\
+M A_{r}-\frac{M B_{\psi}}{a r} \\
a r T_{r r}-2 M T_{\psi r}+\frac{\left(1+M^{2}\right)}{a r} T_{\psi \psi}+\left(a-R_{e} P_{r}\right) T_{r} \\
+M^{\prime} T_{\psi}=-\frac{\operatorname{ar} E_{c} P_{r}}{4 \mu}\left(B^{2}+4 A^{2}\right)
\end{gathered}
$$$$
w=\frac{M^{\prime}}{a r}
$$$$
A=\frac{\mu\left(r M^{\prime}-2 M\right)}{a r^{2}}
$$$$
B=\frac{-4 \mu}{a r^{2}}
$$$$
E=1+M^{2}
$$

$J=a r$

$\cos \alpha=\frac{1}{\sqrt{E}}$

The natural integrability condition $L_{r \psi}=L_{\psi r}$ utilizing equation (39) and (40) yields

$$
\begin{array}{r}
a r A_{r r}-2 M A_{\psi r}-\frac{\left(1-M^{2}\right)}{a r} A_{\psi \psi} \\
+a A_{r}-M^{\prime} A_{\psi}-\left\lceil B_{r}-\frac{f^{\prime} B_{\psi}}{a}\right\rceil_{\psi} \\
=R_{e} w_{r}+R_{e}\left(F_{1}+M F_{2}\right)_{\psi}-R_{e}\left(a r F_{2}\right)_{r}
\end{array}
$$

Once we discover a solution of equation (48), the generalized energy function $L$ and temperature distribution $T$ are determined from equations (39-41). By back substitution we can find the viscosity $\mu$ from either equation (43) or equation (44), the velocity components from (7), the pressure $p$ from (9), and streamlines from (2).

The compatibility equation (48) involves the body force components $F_{1}, F_{2}$ and functions $A$ and $B$ which depends upon the viscosity function $\mu, f(r)$ and derivatives of $f(r)$ which in general are extremely difficult to solve analytically, however we indicated in [19] that the equation resulting from compatibility condition provide solutions on eliminating $\mu$ from the function $A$ and $B$. Following [19] we eliminate $\mu$ from equation (43) and equation (44) by introducing function $X(r)$ through

$A=X(r) B$ where

$X(r)=\frac{-1}{4}\left(r M^{\prime}-2 M\right)$

provided $\left(r M^{\prime}-2 M\right) \neq 0$.

Inserting equation (49) in equation (48), we get

$$
\begin{array}{r}
a r X B_{r r}-(1+2 M X) B_{\psi r} \\
+\frac{M-X\left(1-M^{2}\right)}{a r} B_{\psi \psi}+a B_{r}\left(2 r X^{\prime}+X\right) \\
-B_{\psi}\left(2 M X^{\prime}+M^{\prime} X\right)+a B\left(r X^{\prime}\right)^{\prime} \\
=R_{e}\left(\frac{M^{\prime}}{a r}\right)^{\prime}+R_{e}\left(F_{1}+M F_{2}\right)_{\psi}-R_{e}\left(a r F_{2}\right)_{r}
\end{array}
$$

In equation (51) the coefficients of the derivative $B_{r r}, B_{r \psi}, B_{\psi \psi}, B_{r}, B_{\psi}$ and $B$ are all functions of $r$ only, this suggests to seek a solution of equation (51) of the form

$B(r, \psi)=R(r)+S(\psi)$

Equation (51), on substituting (52), becomes

$$
\begin{array}{r}
\left\{r(X R)^{\prime}\right\}^{\prime}+\frac{M-X\left(1-M^{2}\right)}{a r} S^{\prime \prime} \\
-S^{\prime}\left(2 M X^{\prime}+M^{\prime} X\right)+a\left(r X^{\prime}\right)^{\prime} S \\
=R_{e}\left\{\frac{M^{\prime}}{a r}\right\}^{\prime}+R_{e}\left(F_{1}+M F_{2}\right)_{\psi}-R_{e}\left(a r F_{2}\right)_{r}
\end{array}
$$

Here equation (53) is to provide the function $R(r)$ and $S(\psi)$, but it involves the components of unknown body force $F_{1}$ and $F_{2}$ therefore, its solution will depend upon the form of $F_{1}$ and $F_{2}$. We can select many possible forms of $F_{1}$ and $F_{2}$ leading to the solution of equation (53) for $R(r)$ and $S(\psi)$, however we find that arbitrarily selected forms does not lead to the solution of the momentum equations (3940 ) for the function $L$ and the energy equation (41) for $T$. Our search for the appropriate form of $F_{1}$ and $F_{2}$ revealed that the solution of our equations (39-40) is obtainable if the function $F_{2}$ is a solution of the following differential equation

$$
R_{e}\left(a r F_{2}\right)_{r}=R_{e}\left(\frac{M^{\prime}}{a r}\right)^{\prime}-\left(r(X R)^{\prime}\right)^{\prime}
$$

or

$$
R_{e} F_{2}=R_{e}\left(\frac{M^{\prime}}{a^{2} r^{2}}\right)-(X R)^{\prime}+\frac{G_{1}(\psi)}{a r}
$$

where $G_{1}(\psi)$ is function of integration. On substituting (55) in (53) we find that equation(53) is satisfied for arbitrary $R(r)$ and $S(\psi)$ when $F_{1}$ is

$$
\begin{array}{r}
R_{e} F_{1}=\frac{M-X\left(1-M^{2}\right)}{a r} S^{\prime}-S\left(2 M X^{\prime}+M^{\prime} X\right) \\
+a\left(r X^{\prime}\right)^{\prime} \int S d \psi+H_{1}(r) \\
-M\left[R_{e}\left\{\frac{M^{\prime}}{a^{2} r^{2}}-(X R)^{\prime}+\frac{G_{1}(\psi)}{a r}\right]\right.
\end{array}
$$

where $P_{1}(r)$ is function of integration. Substituting equations (5556), in equation (39-40) and solving for $L$, we have

$$
\begin{aligned}
R_{e} L=\int G_{1}(\psi) d \psi+ & a\left(r X^{\prime}\right) \int S d \psi-(M X+1) S \\
& +e^{r} \int e^{-r} M(X R)^{\prime} d r+e^{r} h_{1}
\end{aligned}
$$


provided

$$
a=1
$$

In equation (57), $R(r)$ and $S(\psi)$ are arbitrary functions and $h_{1}$ is constant of integration.

Utilizing equation (52), the viscosity function is found from equation (43) or (44)

$$
\mu=-\frac{r^{2}}{4}[R(r)+S(\psi)]
$$

The energy equation (41), utilizing equations (49),(52), (58-59) becomes

$$
\begin{array}{r}
r T_{r r}-2 M T_{\psi r}+\frac{\left(1+M^{2}\right)}{r} T_{\psi \psi}+\left(1-R_{e} P_{r}\right) T_{r} \\
-M^{\prime} T_{\psi}=E_{c} P_{r} \frac{\left(1+4 X^{2}\right)}{r}[R(r)+S(\psi)]
\end{array}
$$

The right-hand side of equation (60) suggests seeking solution of the form

$$
T(r, \psi)=R_{1}(r)+R_{2}(r) H(\psi)
$$

Inserting equation (61) in equation (60), we find

$$
\begin{array}{r}
r R_{1}^{\prime \prime}+\left(1-R_{e} P_{r}\right) R_{1}^{\prime}+H(\psi)\left[R_{2}^{\prime \prime}+\left(1-R_{e} P_{r}\right) R_{2}^{\prime}\right] \\
+H^{\prime}(\psi)\left[-2 M R_{2}^{\prime}-M^{\prime} R_{2}\right]+\frac{\left(1+M^{2}\right) R_{2}}{r} H^{\prime \prime}(\psi) \\
=E_{c} P_{r} \frac{\left(1+4 X^{2}\right)}{r}[R(r)+S(\psi)]
\end{array}
$$

Differentiating equation (62) with respect to $\psi$, we get

$$
\begin{array}{r}
H^{\prime}(\psi)\left[R_{2}^{\prime \prime}+\left(1-R_{e} P_{r}\right) R_{2}^{\prime}\right] \\
+H^{\prime \prime}(\psi)\left[-2 M R_{2}^{\prime}-M^{\prime} R_{2}\right]+\frac{\left(1+M^{2}\right) R_{2}}{r} H^{\prime \prime \prime}(\psi) \\
=E_{c} P_{r} \frac{\left(1+4 X^{2}\right)}{r} S^{\prime}(\psi)
\end{array}
$$

Since $r$ and $\psi$ are independent variables therefore the right-hand side of equation (63) demands

$H^{\prime \prime}(\psi)=0$

and

$S^{\prime}(\psi)=s_{1}=$ const.

which implies

$H(\psi)=h_{2} \psi+h_{3}$

and

$S(\psi)=s_{1} \psi+s_{2}$

where $h_{1}, h_{2}$ and $s_{2}$ are constant of integration. Inserting equation (66-67) in equation (63), we get

$$
r R_{2}^{\prime \prime}+\left(1-R_{e} P_{r}\right) R_{2}^{\prime}=\frac{E_{c} P_{r} s_{1}}{h_{1}} \frac{\left(1+4 X^{2}\right)}{r}
$$

Utilizing equation (52), equations (66-67) in equation (62), we get

$$
\begin{array}{r}
r R_{1}^{\prime \prime}+\left(1-R_{e} P_{r}\right) R_{1}^{\prime}=h_{1}\left[2 M R_{2}^{\prime}+M^{\prime} R_{2}\right] \\
-h_{2}\left[r R_{2}^{\prime \prime}+\left(1-R_{e} P_{r}\right) R_{2}^{\prime}\right] \\
+E_{c} P_{r} \frac{\left(1+4 X^{2}\right)}{r}\left[R(r)+s_{2}\right]
\end{array}
$$

when $\left(1-R_{e} P_{r}\right) \neq 0$, the solution of equations (68-69)are

$$
\begin{array}{r}
R_{2}(r)=\int\left[r^{-\left(1-R_{e} P_{r}\right)} \int r^{\left(1-R_{e} P_{r}\right)} Z_{2}(r) d r\right] d r \\
+Q_{1} \int r^{-\left(1-R_{e} P_{r}\right)} d r+Q_{2}
\end{array}
$$

and

$$
\begin{array}{r}
R_{1}(r)=\int\left[r^{-\left(1-R_{e} P_{r}\right)} \int r^{\left(1-R_{e} P_{r}\right)} Z_{1}(r) d r\right] d r \\
+Q_{3} \int r^{-\left(1-R_{e} P_{r}\right)} d r+Q_{4}
\end{array}
$$

where $Q_{1}, Q_{2}, Q_{3}$ and $Q_{4}$ are constant of integration and

$$
\begin{gathered}
Z_{2}(r)=\frac{E_{c} P_{r} s_{1}}{h_{1}} \frac{\left(1+4 X^{2}\right)}{r^{2}} \\
Z_{1}(r)=h_{1}\left[2 M R_{2}^{\prime}+M^{\prime} R_{2}\right]-h_{2}\left[r R_{2}^{\prime \prime}+\left(1-R_{e} P_{r}\right) R_{2}^{\prime}\right] \\
+E_{c} P_{r} \frac{\left(1+4 X^{2}\right)}{r}\left[R(r)+s_{2}\right]
\end{gathered}
$$

Utilizing equations (70-73) in equation(61) we get the temperature $T$ for the case $\left(1-R_{e} P_{r}\right) \neq 0$, then by back substitution we can find the viscosity $\mu$ from equation (59), the velocity components from equation(7), the pressure $p$ from equation(9) using equation(58), and streamlines from equation(2) for arbitrary $f(r)$.

Now when $\left(1-R_{e} P_{r}\right)=0$ the equations (68-69) give

$$
R_{2}(r)=\int\left[\int Z_{2}(r) d r\right] d r+Q_{5} r+Q_{6}
$$

and

$$
R_{1}(r)=\int\left[\int Z_{1}(r) d r\right] d r+Q_{5} r+Q_{6}
$$

Utilizing equations (72-75) in equation(61) we get the temperature $T$ for the case $\left(1-R_{e} P_{r}\right)=0$, then by back substitution we can find the viscosity $\mu$ from equation (59), the velocity components from equation(7), the pressure $p$ from equation(9) using equation(58), and streamlines from equation(2) for arbitrary $f(r)$.

Supplying $M(r)$ from (38) in $\left(r M^{\prime}-2 M\right)=0$, we find that the function $f(r)$ is no more arbitrary but

$$
f(r)=\frac{c_{1} r^{2}}{2}+c_{2}
$$

where $c_{1}$ and $c_{2}$ are constants. For this case the equation (51) becomes

$$
-B_{\psi r}+\frac{M B_{\psi \psi}}{a r}=R_{e}\left(F_{1}+M F_{2}\right)_{\psi}-R_{e}\left(a r F_{2}\right)_{r}
$$


Here equation (77) is to provide the function $B(r, \psi)$ but it involves the components of unknown body force $F_{1}(r, \psi)$ and $F_{2}(r, \psi)$ therefore its solution will depend upon the form of $F_{1}$ and $F_{2}$. We can select many possible forms of $F_{1}$ and $F_{2}$ leading to the solution of equation (77) for $B(r, \psi)$, however we find that all the selected arbitrary forms does not lead to the solution of the momentum equations (39-40) for the function $L$ and the energy equation (41) for $T$. We find that the solution of the equations (39-41) is obtainable if the function $F_{2}$ is a solution of the following differential equation

$$
R_{e}\left(a r F_{2}\right)_{r}=0
$$

or

$$
R_{e} F_{2}=\frac{G_{2}(\psi)}{a r}
$$

where the function of integration is $G_{2}(\psi)$. On substituting equation (79) in (77), we find that arbitrary function $B(r, \psi)$ satisfies equation (77) when the remaining component of the unknown body force is

$$
R_{e} F_{1}=-\frac{c_{1} r G_{2}}{a}-B_{r}+\frac{c_{1} r B_{\psi}}{a}+P_{2}(r)
$$

where the function of integration is $P_{2}(r)$. Utilizing equations (7980 ), in equation (39-40) and solving for the function $L$, we have

$$
\begin{array}{r}
R_{e} L=-\frac{2 c_{1} R_{e} \psi}{a}+\int G_{2}(\psi) d \psi-B(r, \psi) \\
+\int P_{2}(r) d r
\end{array}
$$

The energy equation (41), becomes

$$
\begin{array}{r}
a r^{2} T_{r r}-2 c_{1} r^{3} T_{\psi r}+\frac{\left(1+c_{1}^{2} r^{4}\right)}{a} T_{\psi \psi} \\
+r\left(a-R_{e} P_{r}\right) T_{r}+2 c_{1} r^{2} T_{\psi}=E_{c} P_{r} B(r, \psi)
\end{array}
$$

On substituting values from equation (44) in equation (82), the viscosity $\mu$ is obtained in terms of temperature $T$

$$
\begin{array}{r}
\mu=\left(\frac{-a r^{2}}{4 E_{c} P_{r}}\right)\left[a r^{2} T_{r r}-\right. \\
2 c_{1} r^{3} T_{\psi r}+\frac{\left(1+c_{1}^{2} r^{4}\right)}{a} T_{\psi \psi} \\
\left.+r\left(a-R_{e} P_{r}\right) T_{r}+2 c_{1} r^{2} T_{\psi}\right]
\end{array}
$$

for both the cases when $R_{e} P_{r}=a$ or $R_{e} P_{r} \neq a$, keeping $a$ arbitrary. We can find the velocity components from equation(7), the pressure from equation(9) using equation(81), and streamlines from equation(2) for $f(r)$ given by equation (76).

\section{Results and Discussion}

We have found a class of new exact solutions of the non-dimensional equations governing the two-dimensional steady motion of incompressible fluid of variable viscosity in the presence of body force using following dimensionless parameters

$x^{*}=\frac{x}{L_{0}}, y^{*}=\frac{y}{L_{0}}, u^{*}=\frac{x}{U_{0}}, v^{*}=\frac{v}{U_{0}}$

$\mu^{*}=\frac{\mu}{\mu_{0}}, p^{*}=\frac{p}{p_{0}}, F_{1}^{*}=\frac{F_{1}}{F_{0}}, F_{1}^{*}=\frac{F_{1}}{F_{0}}$

The thermal conductivity $k=k_{0}=$ const.,$\rho=\rho_{0}=$ const. and $c_{v}=c_{p}=$ const . where $c_{v}$ is specific heat at constant volume and $c_{p}$ is specific heat at constant pressure.

The class specifies the stream function characterized by the equation $\theta=f(r)+a \psi+b$ in polar coordinates $r, \theta$, where a continuously differentiable function is $f(r)$ and $a \neq 0, b$ are constants.
The exact solutions are found for given one component of the body force, for both the cases when $f(r)$ is arbitrary and when $f(r)$ is quadratic function of $r$. For arbitrary $f(r)$ the streamlines are $\theta-f(r)-b=\psi=$ Const . and for $f(r)=\frac{1}{2} c_{1} r^{2}+c_{2}$ the streamlines are $\frac{1}{a}\left[\theta-\frac{1}{2} c_{1} r^{2}+c_{2}-b\right]=\psi=$ Const . where $c_{1}$ and $c_{2}$ are constants. In both the cases an infinite set of velocity components, viscosity function, generalized energy function and temperature distribution can be constructed and graph of streamlines can be drawn to observe the streamline patterns. 


\section{References}

[1] Wang, C. Y., On a class of exact solutions of the Navier-Stocks equations, Journal of Applied Mechanics 33 (1966), pp.696-698.

[2] Kapitanskiy, L.V., Group analysis of the Navier-Stokes equations in the presence of rotational symmetry and some new exact solutions, Zapiski nauchnogo sem, LOMI 84(1) (1979), pp.89-107.

[3] Dorrepaal, J. M., An exact solution of the Navier-Stokes equations which describes non-orthogonal stagnation -point flow in two dimensions, Journal of Fluid Mechanics, 163(1) (1986), pp.141-147.

[4] Chandna, O. P.; Oku-Ukpong, E. O., Flows for chosen vorticity functionsExact solutions of the Navier-Stokes Equations,International Journal of Applied Mathematics and Mathematical Sciences 17(1) (1994), pp.155164.

[5] Naeem, R. K., Exact solutions of flow equations of an incompressible fluid of variable viscosity via one - parameter group, The Arabian Journal for Science and Engineering, 19(1) (1994), 111-114.

[6] Naeem, R. K.; Anwer Ali, S., Exact solutions of the equations of motion of an incompressible fluid of variable viscosity ,Karachi University Journal of Science, 24(1) (1996), pp.35-40.

[7] Naeem, R. K.; Srfaraz, A. N., Study of steady plane flows of an incompressible fluid of variable viscosity using Martin's System ,Journal of Applied Mechanics and Engineering, 1(1) (1996), pp.397-433.

[8] Naeem, R. K.; Anwer Ali, S., A class of exact solutions to equations governing the steady plane flows of an incompressible fluid of variable viscosity via von-Mises variables, International Journal of Applied Mechanics and Engineering, 6(1) (2001), pp.395-436.

[9] Naeem, R. K., Steady plane flows of an incompressible fluid of variable viscosity via Hodograph transformation method, Karachi University Journal of Sciences, 3(1) (2003), 73-89.

[10] Naeem, R. K.; Jamil, M., A class of exact solutions to flow equations of an incompressible fluid of variable viscosity, Quaid-e-Awam University Research Journal of Engineering Science and Technology, 6(1,2) (2005), pp.11-18.

[11] Naeem, R. K.; Jamil, M., On plane steady flows of an incompressible fluid with variable viscosity, International Journal of Applied Mathematics and Mechanics, 2(3) (2006), pp.32-51.

[12] Naeem, R. K., On plane flows of an incompressible fluid of variable viscosity ,Quarterly Science Vision, 12(1) (2007), pp.125-131.

[13] Naeem, R. K.; Sobia, Y., Exact solutions of the Navier-Stokes equations for incompressible fluid of variable viscosity for prescribed vorticity distributions ,International Journal of Applied Mathematics and Mechanics, 6(5) (2010), pp.18-38.

[14] Giga, Y.; Inui, K.; Mahalov; Matasui S., Uniform local solvability for the Navier-Stokes equations with the Coriolis force, Method and application of Analysis, 12 (2005), pp.381-384.

[15] Gerbeau, J. -F.; Le Bris, C., A basic Remark on Some Navier-Stokes Equations With Body Forces ,Applied Mathematics Letters, 13(1) (2000), pp.107-112.

[16] Aurnangzeb ;Naeem, R. K., Exact solution to the steady Plane Flows of an Incompressible Fluid of Variable Viscosity Using $(\xi, \eta)$ Coordinates ,World Academy of Science, Engineering and Technology, 3(1) (2009), pp. 839-846.

[17] Naeem, R. K.; Razia S., Some exact solution to the unsteady NavierStokes equations Viscous Incompressible fluid in the presence of Unknown External Force, International Journal of Appl. Math. And Mechanics, 7(11) (2011), pp.83-97.

[18] Mushtaq A., On Some Thermally Conducting Fluids ,Ph. D Thesis,Department of Mathematics, University of Karachi, Pakistan, 2016.

[19] Naeem, R. K.; Mushtaq A., A class of exact solutions to the fundamental equations for plane steady incompressible and variable viscosity fluid in the absence of body force ,International Journal of Basic and Applied Sciences, 4(4) (2015), 429-465. www.sciencepubco.com/index.php/IJBAS. doi:10.14419/ijbas.v4i4.5064

[20] Martin, M. H., The flow of a viscous fluid I ,Archive for Rational Mechanics and Analysis, 14(4) (1971), 266-286.

[21] Weatherburn C. E., Differential geometry of three dimensions ,Cambridge University Press,(1964). 